\title{
HISTORIA
}

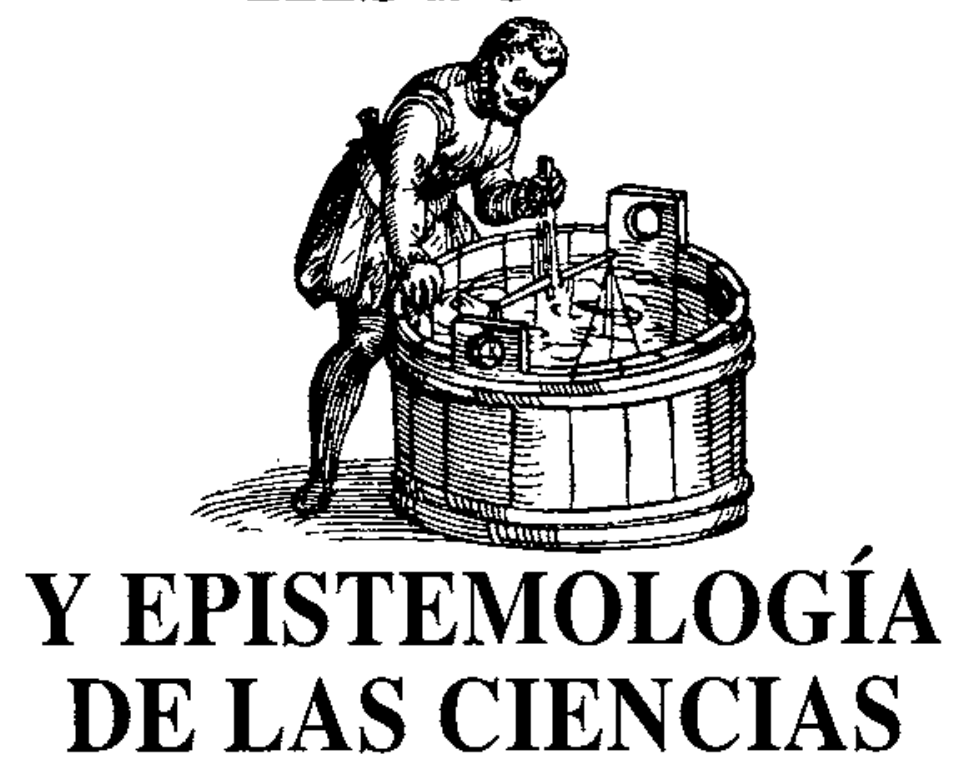

\section{UNA NUEVA FUENTE DE INSPIRACIÓN PARA LA EDUCACIÓN CIENTÍFICA}

LÓPEZ RUPÉREZ, F.

IB Las Rozas. Madrid.

\section{SUMMARY}

In this paper, physics, epistemology and the teaching and learning of physics are presented as a transdisciplinary system. With such an approach, the fundamental principles of contemporary physics considered in a qualitative form -epistemological principles- are postulated as a source of inspiration for Science Education. In this way, a new trend for fundamental educational research is defined.

\section{INTRODUCCIÓN}

La física encarna, hoy más que nunca, los valores del conocimiento humano. Su separación, ya remota, del árbol de la filosofía no ha restado fuerza a su vocación por la comprensión de la totalidad. Identificada con sus orígenes y a la vez reteniendo su vocación filosófica, ha sido capaz de restringir convenientemente los dominios de análisis como una estrategia de avance y ha puesto ent marcha una metodología de resolución de problemas y de construcción de conocimiento cuya característica fundamental es la de disponer de mecanismos autoco- 
rrectivos eficaces en orden a conseguir una representación cada vez más fiable de lo «real». Sin embargo, y más allá de la cuota de progreso que un enfoque de este estilo haya podido aportar a otras disciplinas, la física fundamental alberga en su seno un potencial de conocimiento transponible insuficientemente explotado. No se trata, en este caso, de transportar el segundo principio de la termodinámica al análisis cuantitativo đe los comportamientos sociales, por ejemplo, sino de clevar los principios más generales, característicos de la física contemporánea, de lo cuantitativo a lo cualitativo, de un plano epistemológico más bajo a otro de mayor altura hasta alcanzar una mayor cota de generalidad y, por tanto, un dominio más amplio de aplicabilidad potencial. Detrás de esta empresa se esconde la convicción de la existencia de una cierta unidad fundamental del conocimiento humano que hace plausible la transferencia de significado en su seno de uno a otro ámbito.

Coherentemente con semejante perspectiva, en el presente artículo-de carácter introductorio (López Rupérez 1994)- procederemos en primer Iugar, acotando un conjunto de disciplinas conectadas entre sí e identificándolo como un sistema de carácter transdisciplinar. La física, la epistemología, la enseñanza y el aprendizaje científicos serán sus componentes fundamentales. En segundo lugar, fundamentaremos la posibilidad de transferir significado en el interior de dicho sistema -y mediante procedimientos de corte epistemológico-desde la física fundamental contemporánea a la educación científica.

Frente a otros intentos de inspiración de la investigación sobre la enseñanza y el aprendizaje científicos que han apelado a la psicología o a la filosofía en busca de una adecuada fundamentación disciplinar, la apuesta metateórica que tal enfoque encierra consiste, en definitiva, en conectar aquéllas con ese marco general que para el conocimiento humano ofrece la propia física cuando es leída en clave epistemológica.

\section{SOBRE LA NOCIÓN DE TRANSDISCIPLI- NARIDAD}

El conocimiento científico se ha desenvuelto siempre en la incomodidad de una constante dialéctica entre dos polos extremos. De un lado, la búsqueda de una visión deliberadamente restringida, ajustada a una problemática dada, le ha permitido conseguir niveles cada vez más altos de especificidad en la correspondiente representación de parcelas de lo reaI, haciendo posible la indiscutible eficacia característica de esa forma de conocimiento humano. De otro Iado, la ciencia no ha renunciado jamás al ideal de la unifícación, a la quimera de esa perspectiva global, deseada y deseable, que permitiría captar el conocimiento como un todo coherente en el que quedarían integrados, armónicamente, sus elementos fundamentales.

Esa tensión del espíritu humano entre el conocimiento del todo y el conocimiento de las partes, junto con el correspondiente análisis de Ias posibilidades de integración de ambas perspectivas, ha generado algunos conceptos específicos cuyas resonancias en el ámbito de la educación resultan incuestionables. Me estoy refiriendo a las nociones de multidisciplinaridad, interdisciplinaridad y transdisciplinaridad. Dada la pluralidad de significados que se han atribuido a cada uno de tales términos (Scurati et al. 1977), tiene sentido dedicar algunas líneas a precisar el valor semántico que tendrá para nosotros cada uno de ellos, con una referencia especial a la noción de transdisciplinaridad.

El orden más arriba respetado se corresponde con la dirección de un vector de integración disciplinar creciente. Así, el enfoque de un problema es multidisciplinar cuando incorpora a su resolución aportaciones procedentes de distintas disciplinas pero sin que, en el proceso, cada una de ellas pierda su individualidad, su parcela de competencia, por lo general, bien definida de antemano. Cuando se da un paso más hacia la integración de modo que se producen entre Ias disciplinas intercambios mutuos que inspiran métodos, modelos o estructuras comunes y que concluyen en la aparición de una especie de «hfbridos disciplinares", estamos ante la interdisciplinaridad. En el último escalón de la integración ya anunciada se sitúa la transdisciplinaridad. Frente a la existencia de estructuras o de métodos concurrentes o incluso análogos, característica del peldaño anterior, el enfoque transdisciplinar supone postular la existencia de significados profundos compartidos por un conjunto de disciplinas que pueden circular de unas a otros configurando una red o sistema omnicompresivo. Debido al estatus epistemológico elevado de ese elemento unificador que constituye el significado, las transferencias que puedan producirse entre los diferentes componentes del referido sistema constituyen elementos potenciales de progreso científico en el ámbito restringido de cada una de las disciplinas consideradas.

\section{EL SISTEMA FÍSICA, EPISTEMOLOGÍA Y EDUCACION CIENTIFICA Y SU CARACTER TRANSDISCIPLINAR}

La física, la epistemología -entendida en un sentido amplio- y la educación científica constiłuyen disciplinas cuyas relaciones mutuas, dos a dos, han sido implícita o explícitamente reconocidas desde ámbitos diversos, si bien de un modo aislado. Sin embargo, es posible reclamar para la física, la epistemología, la enseñanza y el aprendizaje científicos el estatus de sistema transdisciplinar y tratar de extraer de ello alguna de sus provechosas consecuencias.

La figura adjunta muestra, mediante un conjunto de grafos, Ias relaciones que entre los diferentes componentes de dicho sistema se postulan. Es evidente que cada uno de sus elementos goza de autonomía suficiente para poder ser reconocido como tal; sin embargo, dicha autonomía se pierde cuando se eleva el plano epistemológico de análisis. En ese nivel más general, cada parte aparece 
conectada con el todo de un modo sustancial. Recurriendo de nuevo al apoyo que facilita el esquema de la figura, procederemos, en lo que sigue, a un análisis más detallado de las relaciones entre los diferentes componentes deI ya referido sistema.

\section{Las relaciones entre la física y su enseñanza}

La ciencia se vierte en su didáctica mediante un conjunto de transformaciones adaptativas que convierten el saber científico en objeto de enseñanza. Esta transposición didáctica (Chevallard 1985) tiende a respetar la estructura de la disciplina y aporta, por tanto, a la enseñanza la componente lógica de la ciencia. La enseñanza interviene, â su vez, en la construcción de la ciencia a través de Ia formación de los futuros científicos dotándolos de un bagaje de conocimientos y de destrezas que constituyen una parte importante de su herencia social. Alguien ha dicho que la estructura conceptual de la ciencia no es otra que la estructura cognitiva que reside en la mente de los grandes científicos (Shavelson 1974).

La enseñanza es esencialmente transferencia, aunque no simple transmisión de conocimiento; por tal motivo, y particularmente en las etapas preliminares de la formación científica, constituye el elemento clave que hace posible la componente acumulativa del quehacer científico. En fases posteriores asociadas a una mayor madurez personal y profesional, la enseñanza de la ciencia es sustituida por la comunicación científica mediante la cual la información relevante circula en el seno de la correspondiente comunidad, soportada en revistas especializadas, reuniones y congresos, pero conservando intactos una buena parte de los elementos claves de aquélla, de sus intenciones y de sus propósitos genéricos.

\section{La enseñanza y el aprendizaje de la física}

Tal y como se ha presentado anteriormente, la enseñanza, entendida en un sentido amplio, constituye un elemento mediador entre la disciplina y su aprendizaje en la mente del sujeto. En un sentido estricto, Ia enseñanza puede identificarse con el acto profesoral por excelencia, con la acción docente que se hace efectiva habitualmente en el aula. Sin embargo, esa noción testringida puede ampliarse hasta identificarse con el concepto de instrucción que incluye la acción de todos los elementos curriculares previstos, sea por un profesor, sea por una institución, para promover el aprendizaje de los alumnos.

El aprendizaje de la física se beneficia de la noción general de aprendizaje en cuanto cambio de conducta de un organismo como resultado de una experiencia anterior (Novak 1982). En el caso de esa modalidad de aprendizaje intelectual se trata de una transformación en la mente del sujeto que lo capacita para incorporar nuevos contenidos cognitivos, para realizar nuevas tareas. La concepción del aprendizaje como logro
(Hewson et al. 1988), como producto de una interacción mediada entre disciplina y sujeto, no excluye en absolu. to el interés por la naturaleza de ese proceso de transformación o cambio cognitivo que Io hace posible. En este marco conceptual, las relaciones recíprocas entre la enseñanza y el aprendizaje de la física se hacen eviden. tes. La enseñanza de la física, entendida en un sentido amplio, facilita el aprendizaje de la disciplina e, inversamente, un buen conocimiento de las claves de un aprendizaje científico efectivo permitirá mejorar la enseñanza de la física, sus contenidos y sus procedimientos. La conexión causal entre enseñanza y aprendizaje no es, pues, de tipo lineal sino más bien circular. Estamos, de hecho, ante dos realidades interactuantes en cuyo seno no se puede establecer una jerarquía bien definida sino que la mejora en uno de esos dos polos de la interacción se produce a través de la del otro y viceversa. Tal circunstancia justifica el bucle que conecta recíprocamente la enseñanza y el aprendizaje de la física en la representación del sistema descrito en la figura 1.

\section{Figura 1}

Representación dẹl conjunto formado por la epistemología, la física, su enseñanza y su aprendizaje. Las relaciones entre los diferentes clementos-que se justifican en el texto- parecen indicat que cstamos ante un sistema transdisciplinar en el seno del cual puede producirse una transfercncia o circulación de significado entrc las partes que mejore su comprensión y su propia fusdamentación.

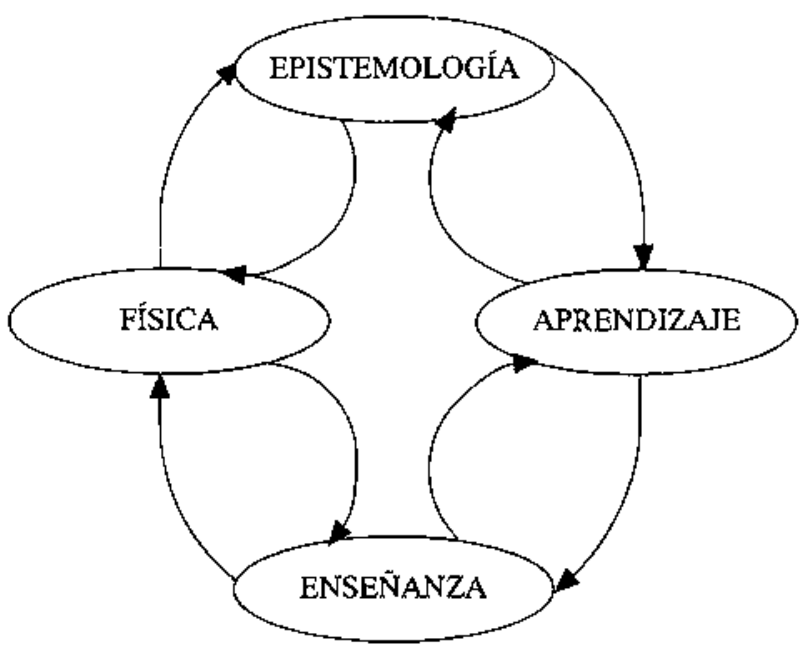

Las relaciones entre epistemología y aprendizaje científico

La ciencia aporta su estructura, sus métođos, sus fundamentos y su historia como objeto del análisis epistemológico. Pero lejos de construir una reflexión pura, la epistemología influye tanto en la enseñanza como en el aprendizaje científicos, lo que conlleva una retroalimentación, por vía indirecta, sobre la propia ciencia. En lo que sigue, centraremos la atención en el análisis de las relaciones entre epistemología y aprendizaje científico (López Rupérez 1990). 
Algunos epistemólogos, que realizaron su obra mucho antes de que se pudiera hablar de una comunidad científíca en didáctica de las ciencias, apostaron por este tipo de relaciones. Tal es el caso del insuficientemente citado Bachelard; su análisis crítico del desarrollo del espíritu científico le permitió avanzar orientaciones en relación con la enseñanza/aprendizaje de las ciencias, las cuales, en una etapa como la presente de auge constructivista, gozan de plena actualidad (Bachelard 1948). Todo ello viene a corroborar la fecundidad potencial de la reflexión epistemológica y, a un tiempo, las raíces filosóficas de esa nueva corriente de investigación en la educación cientifica. Por otra parte, la obra de Piaget ha reforzado las conexiones entre epistemología y aprendizaje científico. Aun cuando Piaget no prestó demasiađa atención a lo que él mismo denominó aprendizaje en sentido estricto (Piaget 1959), el desarrollo de su programa đe investigación sobre epistemología genética pone de manifiesto su interés por el aprendizaje en sentido amplio, es decir, como progreso de las estructuras cognitivas mediante mecanismos de equilibración (Pozo 1987). Sin prescindir del método histórico-crítico en el análisis de la evolución de la ciencia y del ideal científico, Piaget introduce el método psicogenético que procura una explicación causal de los mecanismos intelectuales analizando su formación (Blanche 1972). Con esta original aportación piagetiana un esquema simple de relaciones entre epistemología y aprendizaje científico se hace circular: la epistemología concierne al aprendizaje y está concernida por él. Otros elementos del pensamiento de Piaget, como su visión constructivista del aprendizaje y su valoración del análisis crítico de la historia de la ciencia en calidad de guía adecuada para orientar su didáctica (Piaget et al. 1981), no han hecho más que contribuir a esa conexión recíproca.

Posteriormente, el desplazamiento experimentado en la psicología del pensamiento de la forma al contenido, de la sintaxis a la semántica o de los procesos de razonamiento a la representación (Pozo 1987), ha ejercido, sin duda, una influencia decisiva en la investigación sobre la enseñanza/aprendizaje de las ciencias. En uno y otro caso se ha recurrido explícitamente a la llamada nueva filosofía de la ciencia (Kuhn 1970 a, b, Toulmin 1972, Lakatos 1983) en busca de inspiración (Pozo 1987, López Rupérez 1990). La conexión entre la reflexión sobre la construcción de la ciencia y la reflexión sobre su aprendizaje justifica, pues, por su propia naturaleza, la relación existente entre filosofía de la ciencia y educación científica.

\section{Las relaciones entre física y epistemología}

La existencia de relaciones profundas entre física y epistemología constituye hoy más que nunca un fenómeno incontrovertible. Ei desarrollo de la física modema, al alcanzar los confines de Io «real», ha hecho de la reflexión sobre el alcance del conocimiento y sobre sus fundamentos una tarea necesaria. Un número creciente de físicos téricos de alto nivel científico, procedentes con frecuencia de culturas beneficiarias de una sólida tradición filosófica, son protagonistas de esa nueva orientación. Sirva como ejemplo la declaración que aparece recogida en el primer capítulo del libro La matièreespace-temps de Ios físicos franceses Cohen y Spiro (1990):

Este aparato teórico, este conjunto de conceptos integrados en un formalismo matemático abstracto nos interpela fuertemente y es que las nociones de espacio, de tiempo y de materia tienen un origen a la vez empirico y filosófico. Las convergencias de la física, del empirismo y de la filosofía por aprehender lo que nos rodea traducen la unidad del pensamiento. Ciertas frases o párrafos de este libro podrían emparentarse de igual modo con la filosofía que con la física de particulas; en nuestra opinión la filosofía está presente en la física. Y lo reciproco es también cierto (p. 73).

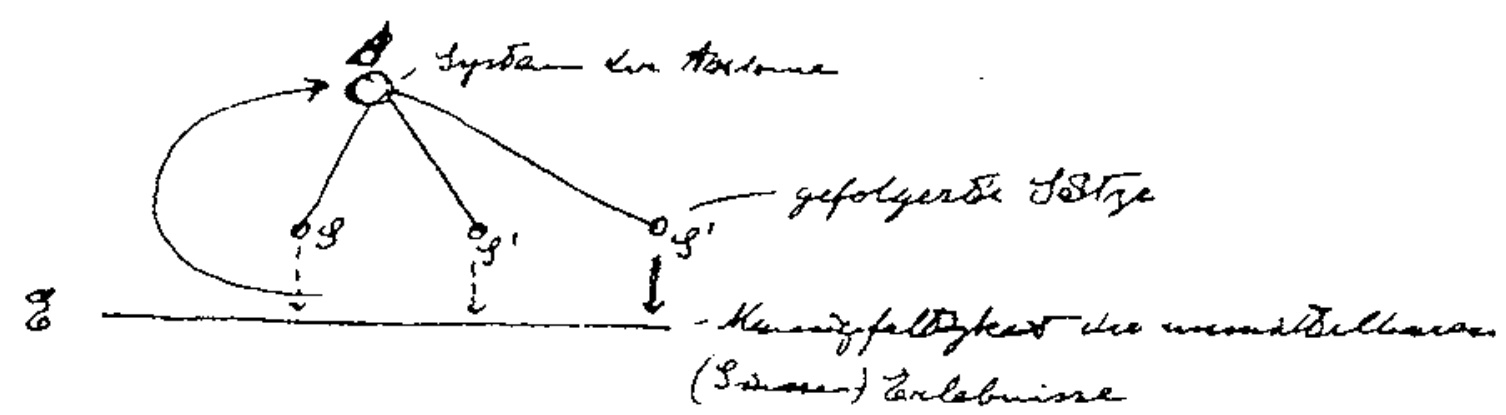


La referida conexión en el sentido física $\longrightarrow$ epistemología se deriva de la propia naturaleza de esta última disciplina que se alimenta de la ciencia y de sus productos aun cuando, a su vez, la enriquezca. Bunge (1985) a la hora de definir la epistemología por su contenido, hace referencia a una lista de ramas tales como: lógica de la ciencia, semántica de la ciencia, teoría del conocimiento científico, metodología de la ciencia, ontología de la ciencia, etc., en la cual la esclarecedora reiteración del genitivo resultaría algo molesta si no fuera estrictamente necesaria. Inversamente, ta influencia de ciertas visiones filosóficas o epistemológicas en los prolegómenos de la gestación de ideas nuevas en la mente de los científicos constituye un hecho relativamente frecuente en la historia de la ciencia (Jammer 1966, Thuiller 1990 $a, b$, Shanahan 1989) y, por tanto, otro elemento de conexión en el sentido epistemologia $\rightarrow$ física anteriormente apuntado. El propio Einstein destacó el carácter bidireccional de dicha conexión en los siguientes términos:

La relación recíproca entre la epistemología y la ciencia goza de una naturaleza destacable. Dependen ambas la una de la otra. La epistemología en ausencia de contacto con la ciencia se convierte en vacía. La ciencia sin epistemología es -aun cuando ello sea solamente pensable-primitiva e intrincada (Einstein 1949, p. 683, citado por Paty 1988, p. 25).

La definición de la física, la epistemología, la enseñanza y el aprendizaje científicos como sistema transdisciplinar se hace, a la luz de esta segunda aproximación, aún más plausible. El análisis en profundidad de sus implicaciones y la explotación de sus consecuencias quedan, por tanto, abiertos sobre el fondo de un horizonte por su propia naturaleza difuso, pero a la vez prometedor.

\section{UNANUEVA FUENTE DEINSPIRACIÓNPARA LA EDUCACION CIENTIFICA}

El avance del conocimiento científico no se produce de un modo lineal. Si bien es cierto que el carácter acumulativo del conocimiento constituye un factor permanente de progreso, no es de ningún modo el único. Blanché (1972), citando a Brunschvieg y Frey, distingue en el ámbito científico entre los progresos de carácter lineal y los de carácter circular $o$, en otros términos, entre los avances de naturaleza progresiva y aquellos otros de carácter reflexivo. Los procesos de carácter circular suponen un ensimismamiento de las propias disciplinas científicas, en sus fundamentos o en la estética de sus construcciones teóricas. En esa tarea, los científicos se alejan del contexto habitual de su trabajo de investigación disciplinar en pos de cotas más profundas de significado, las cuales, una vez alcanzadas, pueden ìluminar el camino hacia niveles de progreso más elevados. La propia noción de revolución científica presenta connotaciones semánticas próximas a lo circular y contiene elementos muy cercanos a los avances de carácter re- flexivo que configuran las etapas o fases preliminares de toda revolución.

La definición de nuestro particular sistema transdisciplinar, por la propia topología de sus relaciones, hace referencia a ese aspecto circular o reflexivo de la elaboración científica. Junto a los bucles de interacción, anteriormente descritos, entre las parejas física-epistemología, epistemología-aprendizaje científico, aprendizaje-enseñanza de la física y física-enseñanza, cabe fijar la atención en ese «anillo externo» (Fig. 1) de relaciones e influencias que constituye un elemento de «cierre» del sistema. De su análisis obtendremos una nueva fuente de inspiración capaz de animar el progreso del referiđo sistema en su conjunto y, particularmente, de los elementos implicados en Ia llamada educación científica.

Por encima de los detalles que conforman la estructura fina de las teorías más características de la física fundamental contemporánea, ningún observador dotado de una mínima sensibiliđad intelectual puede eludir una cierta sensación de plenitud estética que se deriva de la contemplación de la unidad en la diversidad, de la simplicidad de lo complejo y, a un tiempo, de la potencia descriptiva, explicativa y predictiva de esas construcciones teóricas que se integran en un todo armonioso y coherente. Ante una tal situación -y como insinuara ya Bohr con menos elementos de apoyo de los que disponemos en la actualidad-es dificil escapar a la convicción de que los hechos revelados por la teoría cuántica que caen fuera del dominio de nuestras formas ordinarias de intuición proporcionan un medio para elucidar problemas filosóficos de alcance general (Bohr 1927).

El panorama que ofrece la ya referida física fundamental contemporánea -de la cual esa teoría cuántica citada por Bobr es una parte, desde luego importante, pero tan sólo una parte-sugiere que los elementos del conocimiento presentes en ella deben tener una validez más general que la reflejada en sus desarrollos cuantitativos. Bastaría elevar el plano del análisis de lo estrictamente físico a lo propiamente epistemológico para que, desde el ámbito de lo cualitativo, los elementos de conocimiento así designados subtendieran una realidad más amplia que Ia propiamente física. No se trata con ello de resucitar el fisicalismo de Carnap y su tesis de la unidad de Ia ciencia, según la cual toda proposición científica sería expresable en un lenguaje formal, extensión del propiamente físico, el cual estaría llamado a ser la lengua universal de la ciencia (Blanche 1972). La unidad a la que me estoy refiriendo đesplaza su interés de la forma al contenido, de lo sintáctico a lo semántico o, en otros términos, pretende avanzar en pos del significado. Es precisamente buceando en el significado profundo latente en las grandes teorías físicas, por debajo o por encima -según se mire- de su idioma específico, que será posible dar un paso más hacia la consecución đe ese objetivo ideal acariciado igualmente por filósofos y científicos que se encarna en la llamada unidad del conocimiento (Bohr 1964).

La perspectiva antes esbozada sugiere aplicar un procedimiento intelectual de transposición en el seno del 
sistema transdisciplinar más arriba descrito siendo guiados, precisamente, por las indicaciones de los grafos que configuran ese ciclo exterior. Así, una visión cualitativa de ciertos principios muy generales de la física fundamental haría posible -mediante una operación no trivial de transposición de significado-la formulación de otros tantos principios de naturaleza epistemológica que podrían inspirar la investigación sobre la enseñanza y el aprendizaje científicos. Puesto que ese movimiento epistemológico de ascenso y descenso sucesivos nos devuelve al ámbito de lo empírico, la validez de los principios así formulados deberấ ser evaluada por su capacidad a la hora de explicar y predecir fenómenos en el ámbito de la educación científica. Ello demuestra la vocación científica, y no meramente filosófica, de semejante programa. Yendo aún más lejos, una mejora en la eficiencia del proceso de enseñanza-aprendizaje derivada del anterior enfoque ejercerá un efecto retroactivo sobre la propia física como disciplina y sobre el progreso del tipo de conocimiento que le es característico. El bucle se ha cerrado y el carácter transdisciplinar del sistema resulta después de todo, y sí cabe, aún más evidente.

Una vez presentada la anterior perspectiva en sus rasgos fundamentales, tiene pues sentido incidir con algún detenimiento adicional en su legitimidad como procedimiento intelectual y científico. En el orden epistemológico, Ia justificación está, creemos, mínimamente establecida y se deriva, a fin de cuentas, de la aceptación de ese principio de unidad del conocimiento ya referido. Sirva, no obstante, la siguiente cita de Bohr (1970) para reforzar Ia perspectiva general en la que se inserta nuestro planteamiento:

La ampliación del marco conceptual no sólo ha servido para establecer orden en ciertas ramas del conocimiento sino que, en el análisis y sintesis de la experiencia, ha revelado analogias en dominios del conocimiento aparentemente separados, sugiriendo de este modo la posibilidad de una descripción objetiva cada vez más general. En nuestros días la exploración de nuevos campos de experiencia ha revelado condiciones insospechadas previas a toda aplicación no ambigua de nuestros conceptos más elementales, y de este modo ha dado una enseñanza epistemológica cuyo alcance llega a problemas muy alejados de la física (p. 84).

Tiene sentido el bacer explícitos, además, algunos argumentos de legitimación en el orden estético primero y metodológico después. En el orden estético, no cabe duda de que este nuevo enfoque en la inspiración de la educación científica goza de atributos de armonía y coherencia muy parecidos a los que adornan las modernas teorías físicas. La diversidad de los elementos del sistema se resume en una unidad de fondo que añade sentido al significado. Las partes no contribuyen al todo como meros elementos yuxtapuestos sino que influyen en las demás y son influidas por ellas en un marco de relaciones fecundas.

Frente a esta inspiración de la educación científica con base en la física fundamental, las inspiraciones más frecuentes como la de orden psicológico o la de orden filosófico, no parecen alcanzar un nivel similar de armonía y coherencia. No deja de ser curioso que, mientras que la enseñanza de la fúsica rastreaba, en la psicología, la existencia de modelos que inspiraran su progreso, la propia psicología cognitiva acudía a la física en busca de contenidos o formas de conocimiento psicológicamente relevantes. No se pretende, sin embargo, desplazar radicalmente la psicología como elemento facilitador de una mejor comprensión de las características de un aprendizaje científico efectivo, sino más bien de situarla en un nivel más próximo a lo empírico. La psicología cognitiva deberá seguir inspirando, en un proceso «abajoarriba", procedimientos e información observacional relevantes a Ia investigación sobre el aprendizaje de la física pero, en nuestra opinión, ha llegado la hora de intentar apuntar más alto en la búsqueda de principios más generales que iluminen esa otra vía «arriba-abajo» que constituye uno de los elementos esenciales en la construcción del conocimiento que son característicos de la ciencia moderna.

En el orden fijosófico, la inspiración epistemológica de la educación científica ha constituido otro factor fundamental en la evolución de dicha disciplina (Gawthrow y Rowell 1978, Rogers 1982, Hodson 1985, Terhart 1988, López Rupérez 1990, Gil Pérez 1993). Dejando a un lado el elemento de naturaleza historicista, el cual, resucitando en cierta manera el principio biológico de Haeckel -según el cual la ontogenia es una recapitulación de la filogenia-, ha buscado en la evolución científico-intelectual del sujeto hitos semejantes a los aportados por el análisis crítico de la historia de la ciencia, cuya cuota de interés no vamos ahora a discutir, lo cierto es que con bastante frecuencia la ya referida inspiración epistemológica de la educación científica ha estado mediada por la interpretación, a través đe una "filosofía externa», de aspectos referentes a las ciencias físicas tales como el análisis de sus métodos o de sus modos de crecimiento.

La legitimación en el orden metodológico de la inspiración de la educación científica que postulamos hunde sus raíces en la visión que sobre la construcción del conocimiento científico aportaron los físicos-filósofos del presente siglo y que no es, en absoluto, ajena a esa evolución del marco conceptual de la física hacia posiciones cada vez más alejadas del mundo sensible. En este contexto hacemos nuestra para esta ocasión la posicion del propio Einstein a favor del libre juego de los conceptos, en cuanto supuestos del pensamiento, como mecanismo de construcción teórica (Einstein 1970). Dicha posición queđó reflejada de un modo esquemático (Fig. 2) en uno de los fragmentos de la carta escrita a su viejo amigo Maurice Solovine (Holton 1981, Miller 1984) y expresada en los siguientes términos:

Yo veo la cuestión esquemáticamente así:

1) Las $\mathrm{E}$ (experiencias sensibles) nos son dadas.

2) A son los axiomas de los cuales extraemos conclusiones. Psicológicamente, estos A reposan sobre los $\mathrm{E}$. No existe ninguna vía lógica que lleve de los $\mathrm{E}$ a los $\mathrm{A}$, sino 
únicamente una conexión intuitiva (psicológica) que es siempre «hasta nueva orden».

3) De los A se deducen, por via lógica, asertos particulares $\mathrm{S}$, deducciones que pueden pretender la exactitud.

4) Los $\mathrm{S}$ son puestos en relación con los $\mathrm{E}$ (prueba de la experiencia).

Einstcin insiste, en definitiva, en el carácter esencialmente constructivo y especulativo del conocimiento científico, aunque sometido ciertamente a la ligadura fundamental de su coordinación con la experiencia. De otra parte, Niels Bohr, en su ensayo El postulado cuántico y el desarrollo reciente de la teoria atómica (Bohr 1927), viene a poner asimismo el acento en esas dos claves del progreso de la física cuando afirma: «La consecución de esta mayor libertad de ideas es la responsable del maravilloso progreso realizado durante la úitima generación que nos ha permitido penetrar aún más en la naturaleza del fenómeno.»

En el presente caso, el juego conceptual que se postula, el elemento especulativo que se defiende, se inspira en una transposición de significado de la física fundamental contemporánea a la investigación sobre la enseñanza y el aprendizaje científicos aceptándose, no obstante, el veredicto ineludible de la correspondencia con el nivel de lo empírico. En esencia, se trata de extender ese

\section{REFERENCIAS BIBLIOGRÄFICAS}

BACHELARD, G., 1948. La formación del espiritu cientifico, $14^{9}$ ed., 1987. (Siglo XXI: Madrid).

BLANCHE, R., 1972. L'Epistémologie. (PUF; París).

BOHR, N., 1927. La teoría atómica y la descripción de la naturaleza. (Alianza Universidad: Madrid 1988).

BOHR, N., 1964. Física atomica y conocimiento humano. (Aguilar: Madrid).

BOHR, N., 1970. Nuevos ensayos sobre fisica atómica y conocimiento humano. (Aguilar: Madrid).

BUNGE, M., 1985. Epistemologia. (Ariel: Barcelona). procedimiento de ascenso constructivista -propio de la f́́sica- a un ámbito más amplio, más comprehensivo. De esta manera, el principio de invariancia, el de comple. mentaridad, el de exclusión o cl de complejidad de lo elemental -entre otros- convenientemente generalizados, esto cs, desplazados del ámbito de lo cuantitativo al de lo cualitativo bajo la forma de principios epistemológicos (Whittaker 1951, Eddington 1958, Nicolescu 1985, López Rupérez 1994), podrían muy bien aportar una nueva luz, una nueva fuente de inspiración, para la construcción de otras formas de conocimiento; en partí cular, para una nueva fundamentación de la educación científica (López Rupérez 1994).

Después de todo, y como anticipara David Bohm, si en el seno de la ciencia se permite la búsqueda de la elegancia matemática, ¿no estará también permitida la búsqueda de la elegancia conceptual?

\section{NOTA}

El presente trabajo ha recibido apoyo económico del Programa de Ayudas a la Investigación Educativa, 1989, CIDE. Ministerio de Educación y Ciencia. 
GAWTHROW, L.R. y ROWELL, J.A., 1978 Epistemology and Science Education, Studies in Science Education, 5, pp. 31.59 .

GIL PÉREZ, D., 1993. Contribución de la historia y de la filosofía de las ciencias al desarrollo de un modelo de enseñanza/aprendizaje como investigación, Enseñanza de las Ciencias, 11(2), pp. 197-212.

HEWSON, P.W. y HEWSON, M.G., 1988. An Appropriate Concepcion of Teaching Science: A View from Studies of Science Learning, Science Education, 72(5), pp. 597-614.

HODSON, D., 1985. Philosophy of Science, Science and Science Education, Studies in Science Education, 12, pp. 25-67.

HOLTON, G., 1981. L'imagination Scientifique. GaHlimard, París. Trad. al francés de Thematic Origins of Scientific Thought: Kepler to Einstein, Cambridge (Mass.) University Press, 1973 y The Scientific Imagination: Case Stuodies Cambridge, Cambridge University Press, 1978. Trad. cast., Ensayos sobre el pensamiento cientifico en la época de Einstein, Alianza Universidad, Madrid, 1982.

JAMMER, M., 1966. The Conceptual Development of Quantum Mechanics. (McGraw Hill; Nueva York).

KHUN, T.S., 1970a. Postscript, en The Structures of scientific resolutions. (University of Chicago Press: Chicago). (Postdata en la estructura de las resoluciones científicas, Fondo de Cultura Económica, México, 1971).

KHUN, T.S., 1970b. Reflections on my critics en Criticism and the growth of knowledge Lakatos y Musgrane (eds.). (Cambridge University Press: Cambridge). (Trad. cast, 1975, Critica y desarrollo del conocimiento, Grijalbo, Barcelona).

LAKATOS, I., 1983. La metodología de los programas de investigación cientifica. (Alianza Editorial: Madrid).

LÓPEZ RUPÉREZ, F., 1990 . Epistemología y didáctica de las ciencias. Un análisis de segundo orden, Enseñanza de las Ciencias, 8(1), pp. 65-74.

LÓPEZ RUPÉREZ, F, 1994. Más allá de las particulasy de las ondas. Una propuesta de inspiración epistemologica para la educación científica. $2^{\circ}$ Premio Nacional de Investigación Educativa, 1992. (CIDE-MEC).

MILLER, A.I., 1984. Imagery in Scientific Thought. (Ed. Birkhäussen: Boston).
NICOLESCU, B., 1985. Nous, la particule et le monde. (Ed. Le Mail: París),

NOVAK, J.D., 1982. Teoría y práctica de la educación. (Alianza Universidad: Madrid).

PATY, M., 1988. La matière derobée. L'appropriation critique de l'objet de la physique contemporaine. (Ed. des Archives contemporaines: Paris).

PIAGET, J., 1959. Apprentissage et connaissance.(PUF: Patís).

PLAGET, J. y GARCIA,R., 1981. Psicogénesis e historia de la ciencia. (Siglo XXI: México).

POZO, J.I., 1987. Aprendizaje de la ciencia y pensamiento causal. (Visor: Madrid).

ROGERS, P.J., 1982. Epistemology and history in the Teaching of School Science, European Journal in Science Education, $4(1)$, pp. $1+10$.

SCURATI, C. y DAMIANO, E. 1977. Interdisciplinaridad y didactica. (Adara: La Coruna).

SHANAHAN, T., 1989. Kant Naturphilosophie and Oersted's discovery of electromagnetism: a reassessment, Studies in History and Philosophy of Science, Sept. pp. 287-305.

SHAVELSON, R.J., 1974. Methods for examining representations of a subjet-matter structure in a student' memory, Journal of Research in Science Teaching, 11(3), pp. 231-249.

TERHART, E., 1988. Philosophy of Science and School Science Teaching, International Journal in Science Education, 10(1), pp. 11-16.

THUILLIER, P., 1990a. ¿Volverá la mecánica cuántica a reencantar el mundo?, Mundo Científico, 88(10), pp. 88-94.

THUTLLIER, P., 1990b. De la filosofía al electromagnetismo: el caso Oersted, Mundo Científico, 102(10), pp. 562-568.

TOULMIN, S. 1972. Human understanding. Vol. I. The Collective Usage and Evolution of Concepts. Princeton University Press, Princeton (Trad. cast. 1977, La comprensión humana I. El uso colectivo y la evolución de los conceptos, Alianza Editorial: Madrid).

WHITTAKER, E., 1951.Eddington's Principle in the Philosophy of Science. (Cambridge University Press: Cambridge). 